\title{
Effects of Magnetovolume and Spin-orbit Coupling in the Ferromagnetic Cubic Perovskite $\mathrm{BaRuO}_{3}$
}

\author{
Young-Joon Song \\ Department of Applied Physics, Graduate School, Korea University, Sejong 339-700, Korea \\ Kwan-Woo Lef* \\ Department of Display and Semiconductor Physics, Korea University, Sejong 339-700, Korea \\ Department of Applied Physics, Graduate School, Korea University, Sejong 339-700, Korea
}

(Dated: August 28, 2018)

\begin{abstract}
$\mathrm{BaRuO}_{3}$ having five different crystal structures has been synthesized by varying the pressure while sintering. Contrary to the other phases being nonmagnetic, the cubic perovskite phase synthesized recently shows an itinerant ferromagnetic character. We investigated this ferromagnetic $\mathrm{BaRuO}_{3}$ using first principles calculations. A few van Hove singularities appear around the Fermi energy, causing unusually high magnetovolume effects of $\Delta M / \Delta a \approx 4.3 \mu_{B} / \AA$ as well as a Stoner instability $[I N(0) \approx 1.2]$. At the optimized lattice parameter $a$, the magnetic moment $M$ is 1.01 $\mu_{B}$ in the local spin density approximation. When spin-orbit coupling is included, the topologies of some Fermi surfaces are altered, and the net moment is reduced by $10 \%$ to a value very close to the experimentally observed value of $\sim 0.8 \mu_{B}$. Our results indicate that this ferromagnetism is induced by the Stoner instability, but the combined effects of the $p-d$ hybridization, the magnetovolume, and the spin-orbit coupling determine the net moment. In addition, we briefly discuss the results of the tight-binding Wannier function technique.
\end{abstract}

PACS numbers: 71.20.Be, 71.20.Dg, 75.50.Cc, 75.80.+q

Keywords: Electronic structure, $\mathrm{BaRuO}_{3}$, Spin-orbit coupling, magnetovolume effects

\section{INTRODUCTION}

Ruthenates, which formally possess tetravalent $4 d^{4}$ $\mathrm{Ru}$ ions, show interesting properties: unconventional superconductivity in $\mathrm{Sr}_{2} \mathrm{RuO}_{4}$, [1] a metal-insulator transition in $\mathrm{La}_{2} \mathrm{Ru}_{2} \mathrm{O}_{7}$ and $\mathrm{Li}_{2} \mathrm{RuO}_{3},[2$, , 3] metamagnetism in $\mathrm{Sr}_{3} \mathrm{Ru}_{2} \mathrm{O}_{7}$, [4] and complicated magnetic behaviors in the double pyrochlore $R E_{2} \mathrm{Ru}_{2} \mathrm{O}_{7}$ ( $R E=$ rareearth element) [5]. In particular, the Ru-based perovskites $\mathcal{A R u O}_{3}(\mathcal{A}=$ alkaline-earth elements or $\mathrm{Pb})$ have been studied extensively for several decades due to their atypical and controversial electronic and magnetic behaviors [6-12]. $\mathrm{SrRuO}_{3}$ is a metallic ferromagnet with $\mathrm{T}_{C}=160 \mathrm{~K}$ and an experimentally observed moment of $\sim 1.6 \mu_{B}$, whereas $\mathrm{CaRuO}_{3}$ is a nonmagnetic metal 13, 14]. $\mathrm{PbRuO}_{3}$ seems to exhibit incipient magnetism, but whether this system is a metal or an insulator at low- $\mathrm{T}$ is still under debate 15, 16]. The crystal structure of $\mathrm{BaRuO}_{3}$ depends strongly on the temperature and pressure during synthesis [17] whereas the other compounds are orthorhombic. Depending on the amount of corner- and face-sharing $\mathrm{RuO}_{6}$, the structures of $\mathrm{BaRuO}_{3}$ are categorized as the four-, six-, and ten-layered hexagonal phases $(4 H, 6 H$, and $10 H$, respectively), in addition to the nine-layered rhombohedral phase $(9 R) 18-22$. As discussed both theoretically and experimentally [12, 18, 23], the $10 H$ and the $9 R$ phases seem to be semi-metallic whereas the rest are nonmag-

*Electronic address: mckwan@korea.ac.kr netic metals. Early in 2008, Jin et al. synthesized the cubic phase using a high $\mathrm{T}\left(\sim 1000{ }^{\circ} \mathrm{C}\right)$ and high $\mathrm{P}(\sim$ $18 \mathrm{GPa}$ ) technique[17].

In this research, we will focus on the cubic phase, which shows ferromagnetic behavior with $\mathrm{T}_{C}=60 \mathrm{~K}[17]$. On the basis of resistivity measurements, which show a sharp peak in the T-derivation of the resistivity at $\mathrm{T}_{C}$, Jin et al. suggested simple metallic characteristics in the high-T region, but a Fermi-liquid behavior in the low- $\mathrm{T}$ regime. The effective magnetic moment is $2.6 \mu_{B}$, close to the value for $S=1$. However, the saturated moment of 0.8 $\mu_{B}$ is significantly less than the value corresponding to $S=1$, implying atypical magnetic behaviors. Moreover, when pressure is applied, $T_{C}$ decreases, reaching $50 \mathrm{~K}$ at $P=3 \mathrm{GPa}$.

The elastic properties and the pressure effects have been theoretically investigated to some extent 24, 25], but no detailed studies of the magnetic and electric properties of this cubic phase have been reported yet. Thus, we will address the magnetic properties of this system through first-principles calculations, including the local spin density approximation (LSDA), the LSDA plus spinorbit coupling (LSDA+SOC), the fixed spin moment (FSM), and a tight-binding Wannier analysis.

\section{STRUCTURE AND METHOD OF CALCULATION}

First, we optimized the lattice parameter $a$ in the cubic phase. LSDA calculations lead to our optimized $a=3.938 \AA$. This value is about $1.7 \%$ smaller than the 


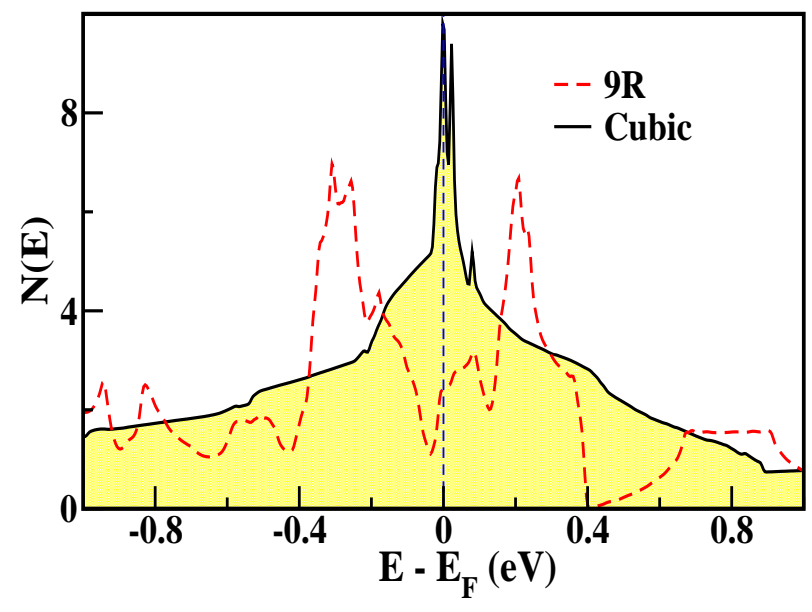

FIG. 1: (Color online) Comparison of the nonmagnetic total densities of states (DOSs) per formula unit between cubic and $9 R \mathrm{BaRuO}_{3}$. For the cubic phase, the optimized lattice parameter was used. In the cubic phase, two sharp peaks appear around the Fermi energy $E_{F}$, resulting in a strong magnetic instability. The vertical dashed line indicates $E_{F}$, which is set to zero.

experimentally-observed value at room temperature 17 . We used the optimized lattice parameter in our calculations, since the total moment at the optimized $a$ is close to the experimental value, as will be discussed in the next section.

Our calculations were carried out with the LSDA and the LSDA+SOC implemented in the all-electron fullpotential code WIEN2k[26]. The basis size was determined by using $\mathrm{R}_{\operatorname{mt}} \mathrm{K}_{\max }=7$ and augmented plane wave sphere radii of 2.5 for $\mathrm{Ba}, 2.0$ for $\mathrm{Ru}$, and 1.7 for $\mathrm{O}$. The Brillouin zone was sampled with a $14 \times 14 \times 14 k$-mesh. For both the LSDA+SOC and the FSM calculations 27], a much denser $k$-mesh of $20 \times 20 \times 20$ was used. The lattice parameter was relaxed until the forces were smaller than $2 \mathrm{mRy} /$ a.u. Furthermore, the all-electron full-potential local-orbital code FPLO was used for the tight-binding Wannier function analysis 28.

\section{RESULTS}

Figure 1 shows the overlapped total densities of states (DOSs) between the nonmagnetic $9 R$ and cubic phases in the range of -1 to $1 \mathrm{eV}$. The DOS of the cubic phase is five times larger than that of the $9 R$ phase due to the sharp peaks at $E_{F}$ and $30 \mathrm{meV}$, suggesting strong magnetic instability.

Using the LSDA, we calculated the change in the total magnetic moment while varying $a$ from the experimental value to a value slightly smaller than the optimized one. As shown in the inset of Fig. 2, the magnetic moment decreases linearly with a slope of $\Delta M / \Delta a \approx 4.3 \mu_{B} / \AA$, indicating strong magnetovolume effects. This value is much larger than that of $\sim 1 \mu_{B} / \AA$ in the Invar systems

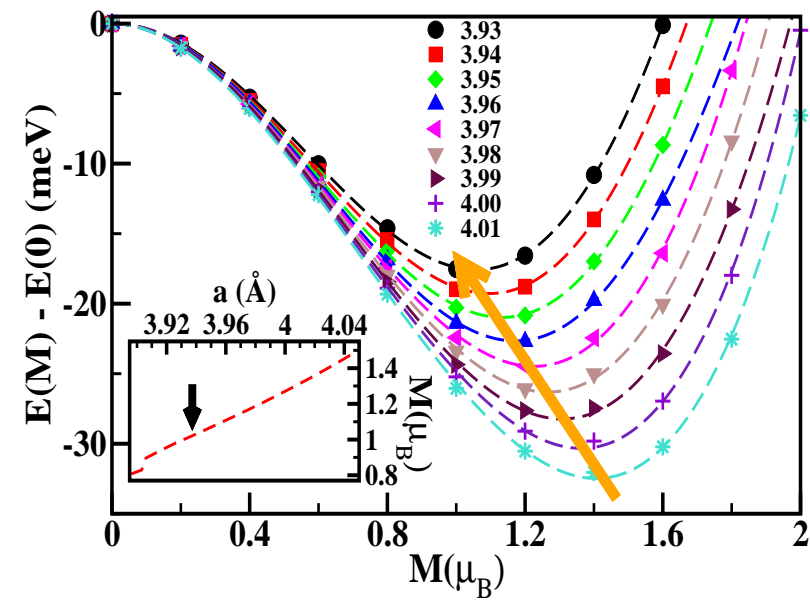

FIG. 2: (Color online) FSM calculations in the cubic phase, while changing the lattice parameter $a$ (in units of $\AA$ ). $E(0)$ denotes the energy of the nonmagnetic state for each $a$. The arrow roughly connects the minimum energy states. Inset: Change in the total magnetic moment $M$ with respect to $a$ in the FM calculations. The arrow indicates the value at the optimized $a$. A discontinuity occurs at $a \sim 3.91 \AA$, because $E_{F}$ pins a van Hove singularity in the spin-up channel (see text).

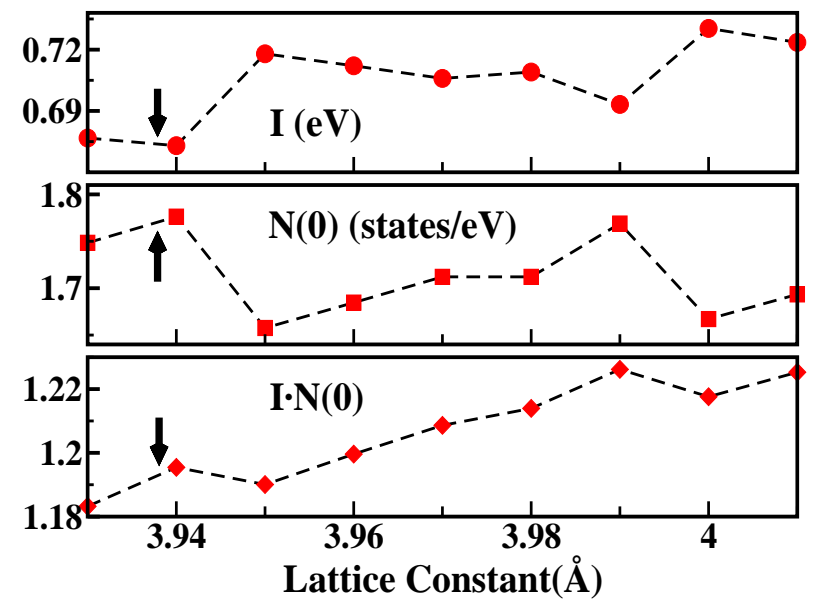

FIG. 3: (Color online) Change in the Stoner parameter $I$ (top), the DOS $N(0)$ per single spin at $E_{F}$ (middle), and $I N(0)$ (bottom) with the lattice parameter in the cubic $\mathrm{BaRuO}_{3}$. Roughly, $I N(0)$ decreases linearly as the lattice parameter decreases.

$\mathcal{A} \mathrm{Fe}_{2}(\mathcal{A}=\mathrm{Y}, \mathrm{Zr}, \mathrm{Hf}$, and $\mathrm{Lu})[29]$.

To clarify these behaviors, FSM calculations were conducted. Plots of energy versus total magnetic moment $M$ for varying values of $a$ are shown in Fig. 2. The difference in energy between the $M=0$ and the ferromagnetic (FM) ground states decreases monotonically from $\sim 32 \mathrm{meV}$ at $a=4.01 \AA$ to $17 \mathrm{meV}$ at $3.93 \AA$. The curves are fitted well by $E(M)-E(0) \approx-\alpha M^{2}+\beta M^{4}$, with constants $\alpha$ and $\beta$, in the low- $M$ region. For the bare susceptibility $\chi_{0}=2 \mu_{B}^{2} N(0)$, the Stoner-enhanced susceptibility is 


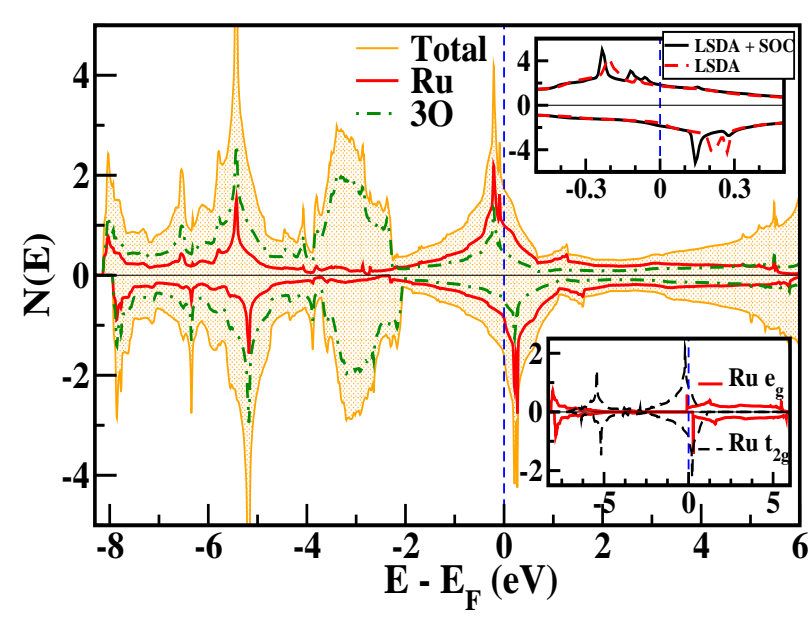

FIG. 4: (Color online) Total and atom-projected DOSs of the FM cubic $\mathrm{BaRuO}_{3}$ in the full range, including all $\mathrm{O} 2 p$ and $\mathrm{Ru} 4 d$ states. In the spin-up channel, peaks appear at -0.2 and $-0.1 \mathrm{eV}$. The DOS $\mathrm{N}(0)$ at $E_{F}$ is 3.34 states per eV for both spins. Upper inset: Expanded view of the total DOSs in LSDA and LSDA+SOC between -0.5 and $0.5 \mathrm{eV}$. Lower inset: $\mathrm{Ru} t_{2 g}$ and $e_{g}$ orbital-projected DOSs.

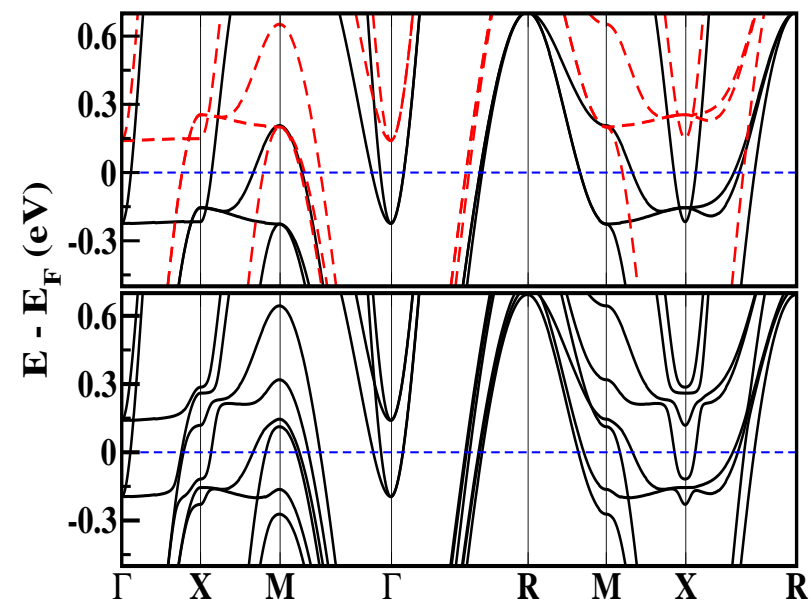

FIG. 5: (Color online) Blowup of the FM band structure in LSDA (top) and LSDA+SOC (bottom) near $E_{F}$ in the cubic $\mathrm{BaRuO}_{3}$. In the top panel, the solid and the dashed lines represent the spin-up and -down characters, respectively. Around the $X$ and the $M$ points, the effects of spin-orbit coupling are substantial. The horizontal dashed lines indicate $E_{F}$.

given by $\chi=\chi_{0} /[1-N(0) I]$, where $N(0)$ is the singlespin DOS at $E_{F}$. In the low- $M$ region, $\alpha=\frac{1}{2} \chi^{-1}$. The top panel of Fig. 3 displays the obtained Stoner parameters $I \approx 0.70( \pm 0.02) \mathrm{eV}$ for each $a$. As $a$ changes, the DOS at $E_{F}$ fluctuates substantially owing to van Hove singularities (vHSs) near $E_{F}$ (see below), as given in the middle panel of Fig. 3, $I N(0)$ decreases monotonically as $a$ decreases, which is consistent with the substantial magnetovolume effects, but for all the lattice parameters studied here, these values exceed the Stoner instability criterion $I N(0) \geq 1$.
Like the FSM results, the LSDA results indicate that the FM state is energetically favored over the nonmagnetic state by $16.4 \mathrm{meV}$ for the optimized lattice parameter. For comparison, from a simple Stoner instability, the energy gain of $\mathrm{Im}^{2} / 4$ for $I \approx 0.7 \mathrm{eV}$ is $175 \mathrm{meV}$, much larger than the calculated value.

Now, we will address the electronic structure of the FM states. Figure 4 shows the total and the atom-projected DOSs, which can be figured out well using the concept of $\mathrm{RuO}_{6}$ cluster orbitals [7]. The $\mathrm{Ru} t_{2 g}$ states, hybridized with the $\mathrm{O} p_{\pi}$ states, produce an antibonding manifold at -2 to $1 \mathrm{eV}$, a bonding manifold at -6 to $-4 \mathrm{eV}$, and a nonbonding manifold at -4 to $-2 \mathrm{eV}$, as can be seen in the lower inset of Fig. 4. The bonding and the antibonding states of the $\mathrm{Ru} e_{g}$ and the $\mathrm{O} p_{\sigma}$ states lie in the regions of -8 to $-6 \mathrm{eV}$ and 0 to $6 \mathrm{eV}$, respectively. The exchange splitting of the $t_{2 g}$ manifold is about $0.5 \mathrm{eV}$. In the FM state, the total moment of $1.01 \mu_{B}$ is decomposed into $0.6 \mu_{B}$ for $\mathrm{Ru}$ and $0.28 \mu_{B}$ for $3 \mathrm{O}$. (The remnant is in the interstitial region.)

The enlarged FM band structure in the LSDA is given in the top panel of Fig. 5. In this region, the spin-up and -down structures are nearly identical, except for a difference in the on-site energy of roughly $0.5 \mathrm{eV}$ due to exchange splitting. In the spin-up channel, in addition to a flat band at $-0.2 \mathrm{eV}$ along the $\Gamma-X$ line, which commonly appears in conventional perovskites, two saddle points, one each at the $X$ and the $M$ points, occur, resulting in vHSs just below $E_{F}$. These features may cause the unusual electrical properties observed experimentally [17].

The band structure of the $t_{2 g}$ and the $p_{\pi}$ clusters in the range of $-8 \mathrm{eV}$ to $2 \mathrm{eV}$ was reproduced well using the tight-binding Wannier function technique. We obtained two important parameters: $p d \pi$ hopping $t_{\pi}=1.23 \mathrm{eV}$ and direct oxygen-oxygen hopping $t_{\pi}^{\prime}=0.17 \mathrm{eV}$. Compared with the tight-binding parameters of the nonmagnetic $\mathrm{CaRuO}_{3}$ obtained by Mazin and Singh[7], $t_{\pi}$ is about $10 \%$ smaller, but $t_{\pi}^{\prime}$ is about half as large. This is consistent with the $20 \%$ smaller bandwidth of the antibonding $t_{2 g}$ manifold in $\mathrm{BaRuO}_{3}$, implying a stronger magnetic instability.

SOC affects the Fermi surface (FS) topology in isovalent $\mathrm{Sr}_{3} \mathrm{Ru}_{2} \mathrm{O}_{7}$ [30], though its effects are often negligible in $4 d$ systems. To consider the effects of SOC, we conducted LSDA+SOC calculations. As shown in the upper inset of Fig. 4, the changes in the DOS are relatively small, leading to a tiny orbital moment of $-0.013 \mu_{B}$ on the $\mathrm{Ru}$ ion. However, the mixing of states with other spin channel reduces the net moment by $10 \%$, bringing it closer to the experimentally observed value of $0.8 \mu_{B}$ [17]. The net moment is $0.91 \mu_{B}$, with local moments of 0.55 $\mu_{B}$ for $\mathrm{Ru}$ and $0.26 \mu_{B}$ for $3 \mathrm{O}$.

An additional remarkable feature is that vHSs move toward $E_{F}$ in both spin channels. In the spin-up channel, the vHS at $-0.1 \mathrm{eV}$ splits into two peaks whereas two vHSs merge and move to $0.1 \mathrm{eV}$ in the spin down channel. These features reflect changes in the band structure at the $X$ and the $M$ points, as demonstrated in the en- 
larged band structure in the bottom panel of Fig. 5. The strength of the SOC is about $0.1 \mathrm{eV}$ at these points near $E_{F}$, but negligible elsewhere. The SOC alters the FS topology at the $X$-points, in particular, in the intersecting pipe-like and cube-like FSs (not shown here), which are often observed in conventional perovskites[31]. The open pipe-like surface becomes closed whereas the cubelike closed one becomes open.

\section{DISCUSSION AND SUMMARY}

The Fermi-liquid behavior experimentally observed in the low- $T$ region may suggest the presence of correlation effects. Considering that this material is a metallic $4 d$ system, the correlation effects should be weak. In our preliminary calculations using the $\mathrm{LDA}+\mathrm{U}$ method, applying $U$ to the Ru ion shifts the $e_{g}$ manifold up and the $t_{2 g}$ manifold down, so only the intersecting pipe-like FS disappears. To evaluate the importance of the correlation effects, detailed experimental observations are necessary.

In summary, we investigated the magnetic properties of the ferromagnetic cubic perovskite $\mathrm{BaRuO}_{3}$, using various first principles calculations: FSM, LSDA, $\mathrm{LSDA}+\mathrm{SOC}$, and the Wannier function technique. In the nonmagnetic DOS, the cubic phase has a sharp peak pinning at $E_{F}$, yielding $I N(0) \approx 1.2$, beyond the Stoner instability. In the LSDA calculations, a few vHSs appear near $E_{F}$ in both spin channels, resulting in a strong magnetovolume effect of $\Delta M / \Delta a \approx 4.3 \mu_{B} / \AA$, significantly higher than that of the Invar system $\mathcal{A} \mathrm{Fe}_{2}$. The effects of the SOC are substantial near $E_{F}$ around the $X$ and the $M$ points and affect the topology of some of FSs, though the strength of the SOC is negligible in most regions. At the optimized volume, the effects of the SOC reduce the net moment by $10 \%$ from the LSDA value, and it becomes very close to the experimentally-observed moment. Our results show that the Stoner instability produces ferromagnetism, and that the combined effects of the hybridization, the magnetovolume, and the SOC determine the magnetic moment. Furthermore, the unusually strong magnetovolume effects may imply atypical transport properties in this $\mathrm{FM} \mathrm{BaRuO}$, suggesting further questions for experimental research.

\section{Acknowledgments}

This research was supported by Korea University.
[1] Y. Maeno, H. Hashimoto, K. Yoshida, S. Nishizaki, T. Fujita, J. G. Bednorz, and F. Lichtenberg, Nature 372, 532 (1994).

[2] S. K. Malik, D. C. Kundaliya, and R. D. Kale, Solid State Commun. 135, 166 (2005).

[3] A. C. W. P. James and J. B. Goodenough, J. Solid State Chem. 74, 287 (1988).

[4] S. A. Grigera, P. Gegenwart, R. A. Borzi, F. Weickert, A. J. Schofield, R. S. Perry, T. Tayama, T. Sakakibara, Y. Maeno, A. G. Green, and A. P. Mackenzie, Science 306, 1154 (2004).

[5] L. J. Chang, M. Prager, J. Perßon, J. Walter, E. Jansen, Y. Y. Chen, and J. S. Gardner, J. Phys.: Condens. Matter 22, 076003 (2010).

[6] J. M. Longo, P. M. Raccah, and J. B. Goodenough, J. Appl. Phys. 39, 1327 (1968).

[7] I. I. Mazin and D. J. Singh, Phys. Rev. B 56, 2556 (1997).

[8] J. T. Rijssenbeek, R. Jin, Yu. Zadorozhny, Y. Liu, B. Batlogg, and R. J. Cava, Phys. Rev. B 59, 4561 (1999).

[9] A. T. Zayak, X. Huang, J. B. Neaton, and K. M. Rabe, Phys. Rev. B 77, 214410 (2008).

[10] J.-S. Zhou, K. Matsubayashi, Y. Uwatoko, C.-Q. Jin, J.G. Cheng, J. B. Goodenough, Q. Q. Liu, T. Katsura, A. Shatskiy, and E. Ito, Phys. Rev. Lett. 101, 077206 (2008).

[11] C. U. Jung, J. Korean Phys. Soc. 58, 83 (2011).

[12] Y. A. Ying, Y. Liu, T. He, and R. J. Cava, Phys. Rev. B 84, 233104 (2011).

[13] Y. Shirako, H. Satsukawa, X. X. Wang, J. J. Li, Y. F. Guo, M. Arai, K. Yamaura, M. Yoshida, H. Kojitani, T. Katsumata, Y. Inaguma, K. Hiraki, T. Takahashi, and M. Akaogi, Phys. Rev. B 83, 174411 (2011).
[14] L. Klein, J. S. Dodge, C. H. Ahn, J. W. Reiner, L. Mieville, T. H. Geballe, M. R. Beasley, and A. Kapitulnik, J. Phys.: Condens. Matter 8, 10111 (1996).

[15] S. A. J. Kimber, J. A. Rodgers, H. Wu, C. A. Murray, D. N. Argyriou, A. N. Fitch, D. I. Khomskii, and J. P. Attfield, Phys. Rev. Lett. 102, 046409 (2009).

[16] J.-G. Cheng, J.-S. Zhou, and J. B. Goodenough, Phys. Rev. B 80, 174426 (2009).

[17] C.-Q. Jin, J.-S Zhou, J. B. Goodenough, Q. Q. Liu, J. G. Zhao, L. X. Yang, Y. Yu, R. C. Yu, T. Katsura, A. Shatskiy, and E. Ito, P. Natl. Acad. Sci. USA 105, 7115 (2008).

[18] T. Ogawa and H. Sato, J. Alloys Compd. 383, 313 (2004).

[19] P. C. Donohue, L. Katz, and R. Ward, Inorg. Chem. 4, 306 (1965).

[20] J. M. Longo and J. A. Kafalas, Mater. Res. Bull. 3, 687 (1968).

[21] S.-T. Hong and A. W. Sleight, J. Solid State Chem. 128, 251 (1997).

[22] J. G. Zhao, L. X. Yang, Y. Yu, F. Y. Li, R. C. Yu, Z. Fang, L. C. Chen, and C. Q. Jin, J. Solid State Chem. 180, 2816 (2007).

[23] C. Felser and R. J. Cava, Phys. Rev. B 61, 10005 (2000).

[24] Y. Cai, Z.-F. Huang, X. Meng, X. Ming, C. Wang, and G. Chen, Solid State Sci. 13, 350 (2011).

[25] D.-M. Han, X.-J. Liu, S.-H. Lv, H.-P. Li, and J. Meng, Physica B 405, 3117 (2010).

[26] K. Schwarz and P. Blaha, Comp. Mat. Sci. 28, 259 (2003).

[27] K. Schwarz and P. Mohn, J. Phys. F: Met. Phys. 14, L129 (1984).

[28] K. Koepernik and H. Eschrig, Phys. Rev. B 59, 1743 
(1999).

[29] W. Zhang, Ph.D. dissertation, Technical University at Dresden (2007).

[30] A. Tamai, M. P. Allan, J. F. Mercure, W. Meevasana, R. Dunkel, D. H. Lu, R. S. Perry, A. P. Mackenzie, D. J.
Singh, Z.-X. Shen, and F. Baumberger, Phys. Rev. Lett. 101, 026407 (2008).

[31] K.-W. Lee and W. E. Pickett, Phys. Rev. B 80, 125133 (2009). 